\title{
A Combined Analysis in Complementary Progeny Tests: Effects on breeding value accuracies
}

\author{
Eduardo P. Cappa ${ }^{1,2, *}$ and Michael U. Stoehr ${ }^{3}$
}

\author{
1 Instituto Nacional de Tecnología Agropecuaria (INTA), Instituto de Recursos Biológicos, Centro de Investigación en \\ Recursos Naturales, De Los Reseros y Dr. Nicolás Repetto s/n, 1686, Hurlingham, Buenos Aires, Argentina. \\ Phone/Fax: +54 1146210433. \\ 2 Consejo Nacional de Investigaciones Científicas y Técnicas (CONICET), Argentina. \\ 3 British Columbia Ministry of Forests, Lands \& Natural Resource Operations, Tree Improvement Branch, 7380 Puckle Road, \\ Saanichton, BC, V8M 1W4, Canada. \\ * Corresponding author: E.P. Cappa, Phone: +54 114621 0433, E-mail: cappa.eduardo@inta.gob.ar
}

\begin{abstract}
Complementary progeny tests allow for simultaneously ranking parents for their general combining ability (GCA) and within-family forward selection. To do this, progeny tests are established with different types of genetic entries (i.e., half-sib and full-sib seedlings, respectively), and different experimental designs. This study proposes a combined analysis of the GCA and full-sib (FS) tests using the mixed model approach to predict simultaneously the breeding values of grandparents, parents, full-sib families and offspring on the same scale. Moreover, a first order autoregressive spatial mixed model for the GCA tests was also implemented in the combined analysis. Our empirical study in coastal Douglas-fir (Pseudotsuga menziesii (Mirb.) Franco) shows that additional information provided from relatives and the overlap genetic entry among GCA and FS tests via the proposed combined analysis, improves the accuracies of breeding values compared to the non-combined analysis. The improvements in the accuracies of breeding values for backward and forward selections were generally modest. Spatial and combined analyses gave slightly better results than the non-spatial combined model.
\end{abstract}

Keywords: mixed linear model, spatial analysis, backward and forward selections, accuracy of breeding values, Douglas-fir

\section{Introduction}

The most important aspect of practical tree breeding is to reliably select parents (i.e., rank them) and assign an accurate genetic quality estimate to these selections in a relatively short time frame. This is accomplished through efficient testing procedures and proper analysis to ensure the identification of the parents as well as an estimate of their quality (breeding value (BV). According to Namkoong (1979, p. 117), precision of the estimates of the genetic value for parents is related to the number of relatives that are evaluated in the progeny test (i.e., the number of progeny per parent or families), and the higher degree of genetic relationships among the different trees, the more reliable these estimates become. Several simulation studies have been carried to assess the efficiency of selection when first and second generation data sets are combined (Johnson 1998a; Ye et al. 2007). However, these computer simulations showed that the inclusion of the previous generation test data only marginally improved the efficiency of breeding value estimation and selection reliability.

Complementary progeny tests were popular in tree breeding programs (Johnson 1998b) during the last two decades. These tests are structured trials were candidate parents are tested in polycross tests, using an equal amount of pollen from a mix of unrelated males, and, at the same time, mated to produce offspring for forward selection. In a sense, it is a two-step approach. That is, parents are tested for their general combining ability via the polycross test (GCA trials) and at the same time used to produce the next generation for forward selection (full-sib family blocks trials). Therefore, this approach can reduce the time of testing and in turn increase gain over time. Johnson (1998b) summarized additional advantages of complementary testing as follows: 1) accurate GCA estimates for parents, 2) parents are tested as outcrossed individuals as unrelated pollen donors are used, 3) potential for more precise within-family selection in family blocks, and 4) simple data analysis. However, there are also drawbacks: 1) the large 
number of half-sib progenies cannot be used for within family selection (unless pedigree reconstruction is used after the fact), and 2) possible rank changes in parental GCA tests cannot be utilized in the crossing for full-sib seedling production (i.e., if one would like to utilize assortative mating, as the best parents are not yet identified as such at the time of full-sib crossing). Also the individual tree breeding values are predicted in a two-step analysis, separate from the one used to estimate parental breeding values with loss of statistical precision. Moreover, there are no direct breeding values predictions of the full-sib families tested in the full-sib family test, as midparent values from the GCA tests are used to rank these families (e.g. White et al. 2007) and full-sib blocks are generally not replicated (Johnson 1998b).

Best linear unbiased predictions (BLUPs) of breeding values using mixed model approach (Henderson 1984) are the norm in forest tree breeding. An important advantage of the mixed model approach is that it allows to combine analyses across trials with different crosses and field designs (experimental and/or plots). Therefore, the BLUP approach is particularly useful for the analysis of complementary progeny tests where different sources of information are combined. When a mixed model analysis of multi-environment trials (MET) is used, the accuracy of predicted breeding values for parents, and offspring can be more reliable, given that more information from relatives is utilized and due to the overlap of genetic entries among the trials (Hardner et al. 2010). Moreover, MET analyses also allows the quantification of the magnitude of the genotype by environmental interactions if related material is tested (e.g. Costa e Silva et al. 2006; Cappa et al. 2013). Finally, mixed models approaches also have the capability of partitioning the environmental variation into a spatially independent and a spatially dependent two dimensional first order autoregressive (AR1) components (Gilmour et al. 1997) within each site.

Global trends or large-scale variation and/or local trend or small-scale variation are well known in forestry field trials as a result of factors such as variations in soil fertility and depth, moisture gradients, or slope. Many studies using mixed models with a first order autoregressive residuals correlation structure for rows and columns (Gilmour et al. 1997) displayed a consistent reduction in the error variance, an increase in the heritability, and in the accuracy of predicted breeding values, in both single (e.g. Costa e Silva et al. 2001; Dutkowski et al. 2002; 2006) and combined (e.g. Ye and Jayawickrama 2008; Hardner et al. 2010) spatial analysis of forest genetic trials. For example, Ye and Jayawickrama (2008) showed that in 275 first-generation progeny trials of Douglas-fir (Pseudotsuga menziesii (Mirb.) Franco) established in the Pacific Northwest, the spatial autoregressive model, on average, removed from $14 \%$ to $34 \%$ of residual variance due to spatial heterogeneity, which resulted in up to $20 \%$ increase in accuracy of breeding value prediction (ranged from $1 \%$ to $20 \%$ for parents and from $1 \%$ to $25 \%$ for offspring relative to the classical non-spatial model). Moreover, they showed that the spatial analysis of MET data had substantially better model fit (i.e., lower values of Akaike's Information Criterion) and provided more accurate breeding value prediction (from $3 \%$ to $17 \%$ ) than the classical non-spatial MET model.

Using mixed linear models we were able to jointly analyse complementary tests in coastal Douglas-fir British Columbia advanced generation breeding despite the polycross tests and the full-sib blocks having different types of genetic entries and experimental designs (e.g., half-sib seedlings in single tree plots versus full-sib seedlings in 25 -tree family blocks). Furthermore, we were able to make an across site spatial analysis of these complementary tests using a first order autoregressive correlation structure within each site. Our main objective was to evaluate the accuracies of predicted BVs for parents and forward selections using a range of mixed models, including the joint analysis of the polycross and full-sibs tests (i.e., combined analysis) using non-spatial and spatial MET models. Additionally, models and resulting estimates of genetic parameters (heritabilities and genetic correlations) for the non-spatial and spatial combined analysis are also compared with corresponding estimates from the separate analysis of the polycross and full-sibs tests.

\section{Material and Methods}

Two series of breeding and testing were used in this study. The basic design, crossing approach and field testing procedures were the same for both series (Series 1 and 2). The parents tested in the two series were different and there were no parents in common between the series.

\section{Crossing Procedure and Seedling Propagationt}

Most parents for the advanced generation breeding in coastal Douglas-fir were forward selected from 8 annual phases of partial, disconnected diallels planted on a total of 88 sites (Heaman and Woods 1989). A small number of parents in Series 1 were forward selections from Pacific Northwest provenance trials and advanced generation parents from the Weyerhaeuser, WA tree improvement program (Stoehr et al. 2008). As the forward selections from the diallels were related to various degrees, it was decided to establish 8 sublines per series with 8 to 17 parent trees per subline. Due to limited cone production, control crossings took several years to complete. All parents were control pollinated with the same 10-donor mixed pollen. Fullsib crosses were made opportunistically (within the constraints of cone production) within sublines. Reciprocal crosses were combined. Seeds from different years of crossing from the same cross were bulked. A total of 85 and 77 half-sib parents and 119 and 80 full-sib families were tested in Series 1 and Series 2 , respectively.

Seedlings were raised at Cowichan Lake Research Station on Vancouver Island following standard growing procedures for coastal Douglas-fir. Half-sib seedlings were planted on four sites per series (GCA tests) using an incomplete block design (Whitacker et al. 2002) with 15 single-tree plots per incomplete block in 30 (Series 1) and 32 (Series 2) replications. Full-sib block tests (FS tests) were planted as $5 \times 5$ tree plots on two sites per series. Series 1 was planted in 1999 while Series 2 was 
planted in 2003. The percentage of mothers in common between the six tests was at least $58 \%$ (Series 1) and $52 \%$ (Series 2). In all GCA tests, five wind-pollinated families (seed obtained from wild-stand trees) were also planted as controls. All sites were brushed and weeded to keep weed competition to a minimum. Total heights $(\mathrm{cm})$ were measured in each series at age 11 from seed. Additional test site information of each series is found in Table 1.

\section{Statistical Analysis and Model Development}

Five multi-environmental individual-tree mixed models were implemented in each of the two series: three separate analyses including data from the four GCA and the two FS tests (hereafter non-combined analysis), and two combined analyses included the joint data across the GCA and FS tests (hereafter combined analysis).

For Series 1 and Series 2 the following linear model was fitted for the four GCA tests (GCA model):

$$
y=\mu 1+Z_{1} s+Z_{2} r+Z_{3} b+Z_{4} a+e
$$

where $y$ is the vector of phenotype data (observation); $\mu$ is the overall mean; $s$ is the vector of random tests effect; $r$ is the vector of random replicate effects within test, with $r \sim N\left(0, I \sigma_{r}^{2}\right)$, where $\sigma_{r}^{2}$ is the replicate within test variance (a common variance was used) and $\boldsymbol{l}$ is the identity matrix; $\boldsymbol{b}$ is the vector of random incomplete block effects within replication and test, with $\boldsymbol{b} \sim N\left(\boldsymbol{0}, \boldsymbol{I} \sigma_{b}^{2}\right)$, where $\sigma_{b}^{2}$ is the incomplete block within replication and test variance (a common variance was used); $a$ is the vector of random additive breeding values of all individuals of the GCA tests, with $\mathbf{a} \sim N\left(\mathbf{0}, \boldsymbol{G}_{A} \otimes \boldsymbol{A}\right)$, where $\boldsymbol{G}_{A}$ is the additive genetic (co)variance matrix between the GCA tests with diagonal elements $\sigma_{a_{i j}}^{2}$ representing the additive variance of the jth test and off-diagonal elements $\sigma_{a_{i j}}$, representing the additive genetic covariance between test $j$ and $j^{\prime}$, the matrix $A$ contains the additive relationships among all trees of the GCA tests, and $\otimes$ represents the Kronecker product. Finally, $e$ is the random vector of residuals, with $e \sim N(0, R)$, where $\boldsymbol{R}=\oplus \boldsymbol{R}_{j}=\bigoplus \boldsymbol{I}_{n_{j}} \sigma_{e_{j}}^{2}, \sigma_{e_{j}}^{2}$, are scalars representing the error variance for eăch test $j$, with $j=1, \ldots, 4, I_{n_{j}}$ is the identity matrix of dimension equal to the number of observation within each $j$ th test $\left(n_{j}\right)$, and $\oplus$ represents the 'direct sum' of matrices notation. Preliminary ${ }^{j}$ alnalyses indicated that there was considerable spatial heterogeneity within the four GCA tests of both series. Therefore, a spatial autoregressive residual structure was also implemented within each GCA trials by dividing the vector $\boldsymbol{e}$ into spatial dependent $(\xi)$ and spatially independent (n) residuals (e.g., Costa e Silva et al. 2001. Gilmour et al. (1997) suggested to model the $\xi$ vector as a separable Kronecker product of first-order autoregressive covariance structures (AR1) on the rows (row) and the columns (col), i.e., $\sigma_{\xi}^{2}\left[\operatorname{AR} 1\left(\rho_{\text {col }}\right) \otimes \operatorname{AR} 1\left(\rho_{\text {row }}\right)\right]$ where $\sigma_{\xi}^{2}$ is the spatial residual variance, and $\rho_{i}$ the autocorrelation parameter. The vector $\eta$ were assumed pairwise independent, i.e., $I \sigma_{\eta}^{2}$, where $\sigma_{\eta}^{2}$ is the independent residual variance. Hereafter this last model is referred as GCA_sp model. Also, 1 is the vector of ones; and $\mathbf{Z}_{1}, \mathbf{Z}_{2^{\prime}} \mathbf{Z}_{3^{\prime}}$ and $\mathbf{Z}_{4^{\prime}}$ are all incidence matrices for their respective effects.

For both series the following linear mixed model was fitted for the two FS tests (FS model):

$$
y=\mu 1+Z_{1} s+Z_{2} f+Z_{3} a+e
$$

where $y$ is the vector of phenotype data (observation); $\mu$ is the overall mean; $s$ is the vector of random tests effect; $f$ is the vector of random full-sib family genetic effects (confounded with the plot effects), with $\boldsymbol{f} \sim N\left(\boldsymbol{O}, \boldsymbol{G}_{F} \otimes \boldsymbol{I}\right)$, where $\boldsymbol{G}_{F}$ is the family genetic (co)variance matrix between the FS tests with diagonal elements $\sigma_{f}^{2}$ representing the family variance (a common variance was used) and off-diagonal elements $\sigma_{f_{i j^{\prime}}}$ representing the family genetic covariance between test $j$ and $j$;; $a$ is the

Table 1

\begin{tabular}{|c|c|c|c|c|c|c|c|c|}
\hline \multicolumn{9}{|l|}{ Series 1} \\
\hline Local name & Site number & Type of test ${ }^{1}$ & Spacing $(\mathrm{m})$ & Latitude & Longitude & Elevation (m) & \# of test Trees & $\begin{array}{l}\text { Age } 11 \text { Height } \\
\text { Means (SD; cm) }\end{array}$ \\
\hline Buckley & 1 & GCA & $2 \times 2$ & $49^{\circ} 33^{\prime} 63^{\prime \prime}$ & $124^{\circ} 54^{\prime} 46^{\prime \prime}$ & 180 & 2295 & $501.3(117)$ \\
\hline Snowdon ${ }^{2}$ & 2 & GCA & $1 \times 1$ & $50^{\circ} 04^{\prime} 54^{\prime \prime}$ & $125^{\circ} 21^{\prime} 04^{\prime \prime}$ & 96 & 2479 & 579.3 (109) \\
\hline Noomas & 3 & GCA & $2 \times 2$ & $50^{\circ} 23^{\prime} 75^{\prime \prime}$ & $126^{\circ} 54^{\prime} 96^{\prime \prime}$ & 460 & 2167 & $661.0(111)$ \\
\hline Canoe & 4 & GCA & $2 \times 2$ & $49^{\circ} 47^{\prime} 31^{\prime \prime}$ & $124^{\circ} 18^{\prime} 88^{\prime \prime}$ & 171 & 2095 & 810.5 (151) \\
\hline Holiday & 5 & FS & $2 \times 2$ & $49^{\circ} 30^{\prime} 69^{\prime \prime}$ & $124^{\circ} 52^{\prime} 61^{\prime \prime}$ & 105 & 2452 & 440.2 (99) \\
\hline Snowdon ${ }^{2}$ & 6 & FS & $1 \times 1$ & $50^{\circ} 04^{\prime} 51^{\prime \prime}$ & $125^{\circ} 21^{\prime} 00^{\prime \prime}$ & 96 & 2470 & $623.8(124)$ \\
\hline \multicolumn{9}{|l|}{ Series 2} \\
\hline Hillcrest & 1 & GCA & $2 \times 2$ & $48^{\circ} 46^{\prime} 24^{\prime \prime}$ & $124^{\circ} 07^{\prime} 45^{\prime \prime}$ & 210 & 1806 & $677.7(120)$ \\
\hline Big Tree & 2 & GCA & $2 \times 2$ & $50^{\circ} 15^{\prime} 80^{\prime \prime}$ & $125^{\circ} 43^{\prime} 59^{\prime \prime}$ & 225 & 2247 & $528.6(95)$ \\
\hline Jordan River & 3 & GCA & $2 \times 2$ & $48^{\circ} 25^{\prime} 80^{\prime \prime}$ & $124^{\circ} 02^{\prime} 08^{\prime \prime}$ & 145 & 1671 & 729.7 (114) \\
\hline Museeum & 4 & GCA & $2 \times 2$ & $49^{\circ} 05^{\prime} 12^{\prime \prime}$ & $124^{\circ} 40^{\prime} 33^{\prime \prime}$ & 410 & 2055 & $439.3(89)$ \\
\hline Jordan River & 5 & FS & $2 \times 2$ & $48^{\circ} 25^{\prime} 86^{\prime \prime}$ & $124^{\circ} 01^{\prime} 80^{\prime \prime}$ & 160 & 1667 & 803.9 (118) \\
\hline North Arm & 6 & FS & $2 \times 2$ & $48^{\circ} 50^{\prime} 72^{\prime \prime}$ & $124^{\circ} 06^{\prime} 53^{\prime \prime}$ & 168 & 1595 & 762.6 (119) \\
\hline
\end{tabular}

Geographic and test information for six progeny tests of coastal Douglas-fir in each of two series.

NOTE: 1 GCA is general combining ability test, FS is a full-sib test using family blocks. 
vector of random additive genetic effects of individuals (or breeding values) for the FS tests, with $\boldsymbol{a} \sim N\left(\boldsymbol{0}, \boldsymbol{G}_{A} \otimes \boldsymbol{A}\right)$ where $G_{A}$ is the additive genetic (co)variance matrix between the FS tests with diagonal elements $\sigma_{a_{i j}}^{2}$ representing the additive variance of the jth test and off-diagonal elements $\sigma_{a_{i j},}$ representing the additive genetic covariance between test $j$ and $j$, and the matrix $A$ contains the additive relationships among all trees of the FS tests. Finally, $\boldsymbol{e}$ is the random vector of residuals, with $e \sim N(0, R)$, where, $\boldsymbol{R}=\bigoplus \boldsymbol{R}_{j}=\bigoplus \boldsymbol{I}_{n_{j}} \sigma_{e_{j}}^{2}, \sigma_{e_{j}}^{2}$ are scalars representing the error variance $\bar{f}^{1}$ or each test $j$, with $j=1, \ldots, 2$. Also, 1 is the vector of ones; and $Z_{1^{\prime}} Z_{2^{\prime}}$ and $Z_{3^{\prime}}$ are all incidence matrices for their respective effects. Note that there is no spatial model for the non-combined analysis of the two FS tests.

A final combined analysis was done for all tests using the following linear mixed model (GCA+FS model):

$$
y=\mu 1+Z_{1} s+Z_{2} r+Z_{3} b+Z_{4} f+Z_{5} a+e
$$

where $y$ is the vector of phenotype data (observation); $\mu$ is the overall mean; $s$ is the vector of random tests effect; $r$ is the vector of random replicate effects within of each GCA test, with $\boldsymbol{r} \sim N\left(\boldsymbol{0}, \boldsymbol{I} \sigma_{r}^{2}\right)$, where $\sigma_{r}^{2}$ is the replicate within test variance (for the four GCA tests, a common variance was used) and $l$ is the identity matrix; $b$ is the vector of random incomplete block effects within replication and GCA test, with $\boldsymbol{b} \sim N\left(\boldsymbol{0}, \boldsymbol{I} \sigma_{b}^{2}\right)$, where $\sigma_{b}^{2}$ is the incomplete block within replication and test variance (for the four GCA tests, a common variance was used); $f$ is the vector of random full-sib family genetic effects (confounded with the plot effects) for the FS tests, with $\boldsymbol{f} \sim N\left(\boldsymbol{0}, \boldsymbol{G}_{F} \otimes \boldsymbol{I}\right)$, where $\boldsymbol{G}_{\boldsymbol{F}}$ is the family genetic (co)variance matrix between the FS tests with diagonal elements $\sigma_{f}^{2}$ representing the family variance (for the FS tests, a common variance was used) and off-diagonal elements $\sigma_{f_{i j},}$ representing the family genetic covariance between test $j$ and $j$; a is the vector of random additive breeding values of all individuals for all tests, with $\mathbf{a} \sim N\left(\boldsymbol{0}, \boldsymbol{G}_{A} \otimes \boldsymbol{A}\right)$, where $\boldsymbol{G}_{\mathrm{A}}$ is the additive genetic (co)variance matrix between the six tests with diagonal elements $\sigma_{a_{i j}}^{2}$ representing the additive variance of the $j$ th test and off-diagonal elements $\sigma_{a_{i j^{\prime}}}$ representing the additive genetic covariance between test $j$ and $j^{\prime}$, and the matrix $A$ contains the additive relationships among all trees of all tests. Finally, $e$ is the random vector of residuals, with $e \sim N(0, R)$, where $\boldsymbol{R}=\bigoplus \boldsymbol{R}_{j}=\overbrace{}^{s} \boldsymbol{I}_{n_{j}} \sigma_{e_{j}}^{2}, \sigma_{e_{j}}^{2}$, are scalars representing the error variance for each test $j$, with $j=1, \ldots, 6$. Additionally, a spatial residual structure (Gilmour et al. 1997) was also implemented within each GCA tests and this model is referred as GCA+FS_sp. Also, 1 is the vector of ones; and $Z_{1^{\prime}} Z_{2^{\prime}} Z_{3^{\prime}} Z_{4^{\prime}}$ and $Z_{5^{\prime}}$ are tall incidence matrices for their respective effects.

Note that the additive genetic correlations $\left(r_{a_{i j^{\prime}}}\right)$ between tests are estimated by the unstructured $G_{A}$ matrix. However, we finally reported the results from a $G_{A}$ matrix with different additive variances for each tests but the same additive genetic covariance between pair of tests (this allows a more parsimonious model).

The ASReml (version 3.0) program was used for all analyses (Gilmour et al. 2009). Given the variance components estimated from each GCA test, single-site narrow sense individual heritabilities $\left(\hat{h}_{j}^{2}\right)$ were estimated as:

$$
\hat{h}_{j}^{2}=\frac{\hat{\sigma}_{a_{j}}^{2}}{\hat{\sigma}_{a_{j}}^{2}+\hat{\sigma}_{e_{j}}^{2}}
$$

where $\hat{\sigma}_{a_{j}}^{2}$ is the estimated additive genetic variance of the $j$ th test, $\hat{\sigma}_{e_{j}}^{2}$ is the estimated error variance of the jth test. For the spatial models (i.e., GCA_sp and GCA+FS_sp models), the $\hat{\sigma}_{e_{j}}^{2}$ was replaced for the estimated independent residual variance of the jth test $\left(\hat{\sigma}_{\eta_{j}}^{2}\right)$ (see for example, Ye and Jayawickrama 2008). The heritabilities of FS tests were not reported because the mating and design of these trials (i.e., unreplicated plots containing trees from a single family) are not designed to obtain heritability estimates.

The BVs were expressed as a percentage of the control trees mean for each series $(597 \mathrm{~cm}$ and $545 \mathrm{~cm}$ for Series 1 and 2 , respectively). For non-combined and combined analyses, BVs from each site were averaged using the heritability of each test as a weight factor to obtain a single BV for each test entry. Thus, more weight was given to the sites with high heritabilities.

Model comparison was provided by the accuracy of prediction of breeding values, which was computed using the following expression:

$$
r=\sqrt{\frac{1-\mathrm{PEV}}{\hat{\sigma}_{a_{j}}^{2}}}
$$

The acronym PEV stands for 'prediction error variance' of predicted BVs using BLUPs of parent and offspring, this was calculated following to Henderson (1984).

Further model comparison of the non-combined (and combined) spatial and non-spatial models was provided by the Akaike Information Criterion (AIC) values (Akaike 1974): AIC $=-2 \times \log \mathrm{L}+2 \times d$, where log $\mathrm{L}$ is the REML log-likelihood for the estimated model and $d$ is a measure of model complexity, "the effective number of parameters". Models having a smaller AIC should be favored, as this indicates a better fit and a lower degree of model complexity. Unfortunately, the combined analysis for both non-spatial and spatial models cannot be compared with the respective non-combined analysis (i.e., GCA+FS model vs. GCA or FS models, and GCA+FS_sp model vs. GCA_sp model) using the AIC, as AIC is not appropriate to compare models based on different data sets. However, we summed the AIC values from the non-combined FS and GCA_sp models and compared these values with those from the combined GCA+FS_sp model.

To study the impact of the combined analysis on the backward and forward selection decisions, further comparisons were provided by Spearman rank correlations. Specifically, Spearman correlations were computed for comparing whether the ranking of predicted breeding values for parents and offspring differ among the non-combined and combined models. Average of predicted breeding values, weighting by the heritability of each site, were used to calculate the Spearman correlations in each Series. For the non-combined models, weighting predicted breeding values of parents were averaged across the 
GCA tests, while weighting predicted breeding values of offspring were averaged across the FS tests. For the combined models, weighting breeding values of parents and offspring were averaged across the six GCA and FS tests.

\section{Results}

\section{Model Comparisons}

When we compared the AIC values from the non-combined FS and GCA_sp models with those from the combined GCA+FS_sp model, the results showed a slight improvement for the combined analysis only in the Series 1 (similar results were obtained using the logL) (Table 2). However, the non-combined and combined spatial MET analyses gave an improvement over the non-spatial MET models in the two series. For Series 1, the AIC criteria of the non-combined (GCA_sp model) and combined (GCA+FS_sp model) spatial MET models were smaller than the respective non-combined (GCA model) and combined (GCA+FS model) non-spatial MET models. The AIC values for Series 2 following the same trend as Series 1, i.e., the non-combined and combined spatial MET models were better than the respective non-spatial MET models (Table 2 ).

\section{Impact of Combined Analysis on Genetic Parame- ters}

In Series 1, small and variable changes in the single-site narrow sense individual heritabilities $\left(\hat{h}^{2}\right)$ between combined and non-combined models were observed (Table 2). However, in Series 2, the estimated $\hat{h}^{2}$ for the combined models were higher than that estimated for non-combined models for both non-spatial (average of $7.6 \%$ ) and spatial (average of $7.1 \%$ ) analyses (Table 2).

The additive genetic correlations $\left(r_{a_{i i^{\prime}}}\right)$ for the combined models were smaller than those obtained from the non-combined models, in both non-spatial and spatial analyses (Table 2). In spite of the family term being confounded with plot effect, the same trend was found for the family genetic correlation $\left(r_{f_{i j^{\prime}}}\right)$, i.e., smaller values were obtained from the combined non-spatial model in the in Series 1 (results not shown). However, both $r_{a_{i j^{\prime}}}$ and $r_{f_{i j^{\prime}}}$ correlation were fairly consistent between the non-spatial (GCA+FS model) and spatial (GCA+FS_sp model) combined analyses.

\section{Impact of Combined Analysis on Accuracy of Predicted Breeding Values}

Table 3 shows the average accuracies of prediction breeding values for all model studied and for parents and offspring at individual site. As expected, average accuracy of prediction breeding values for parents from the non-combined GCA model were higher for the four GCA tests in both series than the respective values for the two FS tests. Meanwhile, average accuracy of prediction breeding values for offspring were higher for the two FS sites than those values for the four GCA sites (Table 3), as full-sibs "sampled" a much narrower range in microenvironments (thus reducing phenotypic error), growing in a contiguous $5 \times 5$ block.

For parents, the average accuracy of breeding values across the six complementary tests from the non-spatial combined MET model (GCA+FS model), were higher than those obtained from the non-spatial non-combined models (GCA and FS models) in both Series studied (Table 3). However, these accuracies varied between the GCA tests and the FS tests. Meanwhile, the average accuracy for parents across the GCA tests from the combined GCA+FS model were slightly smaller for Series $1(-0.67 \%)$ and slightly higher for Series 2 (0.49\%) than those values from the non-combined GCA and FS models, the corresponding values for the FS tests were $8.8 \%$ and 22.59 $\%$. Thus, these results highlight the inclusion of the GCA tests on the accuracies of parents of the FS tests. Averaging across the six complementary tests, the accuracies of BVs for parents

\section{Table 2}

Log-likelihood (LogL), Akaike Information Criterion (AIC) values, additive genetic correlations $\left(\hat{r}_{a}\right)$, and single-site narrow sense individual heritabilities for the general combining ability tests ( $\hat{h}^{2}$; standard error in parentheses) for the two complimentary test series of coastal Douglas-fir progeny tests using the non-combined non-spatial model for the full-sib (FS model) and general combining ability tests (GCA model), the non-combined spatial model (GCA_sp model), and the combined non-spatial (GCA+FS model) and spatial (GCA+FS_sp model) models. See text for a complete description of the models.

\begin{tabular}{|c|c|c|c|c|c|c|c|c|c|c|c|c|}
\hline & \multicolumn{6}{|c|}{ Series 1} & \multicolumn{6}{|c|}{ Series 2} \\
\hline & GCA & Model & & & & & GCA & Model & & & & \\
\hline & SITE & FS & GCA & GCA_sp & $\mathrm{GCA}+\mathrm{FS}$ & GCA+FS_sp & SITE & FS & GCA & GCA_sp & $\mathrm{GCA}+\mathrm{FS}$ & GCA+FS_sp \\
\hline LogL & & -24.38 & -46.68 & -46.12 & -71.05 & -70.48 & & -16.81 & -39.49 & -39.24 & -56.31 & -56.06 \\
\hline AIC & & 48.74 & 93.33 & 92.19 & 142.06 & 140.91 & & 33.61 & 78.96 & 78.44 & 112.58 & 112.06 \\
\hline$\hat{r}_{a}$ & & $0.52(0.13)$ & $0.77(0.07)$ & $0.76(0.07)$ & $0.66(0.06)$ & $0.65(0.06)$ & & $0.39(0.20)$ & $0.81(0.07)$ & $0.81(0.07)$ & $0.69(0.07)$ & $0.70(0.07)$ \\
\hline \multirow{4}{*}{$\hat{h}^{2}$} & 1 & - & $0.14(0.05)$ & $0.19(0.06)$ & $0.14(0.04)$ & $0.19(0.06)$ & 1 & - & $0.30(0.08)$ & $0.30(0.08)$ & $0.34(0.09)$ & $0.34(0.09)$ \\
\hline & 2 & - & $0.40(0.08)$ & $0.43(0.08)$ & $0.40(0.08)$ & $0.43(0.08)$ & 2 & - & $0.29(0.07)$ & $0.36(0.08)$ & $0.31(0.07)$ & $0.38(0.09)$ \\
\hline & 3 & - & $0.23(0.06)$ & $0.23(0.06)$ & $0.22(0.06)$ & $0.23(0.06)$ & 3 & - & $0.23(0.07)$ & $0.23(0.07)$ & $0.24(0.08)$ & $0.25(0.08)$ \\
\hline & 4 & - & $0.22(0.06)$ & $0.24(0.07)$ & $0.21(0.06)$ & $0.24(0.06)$ & 4 & - & $0.27(0.07)$ & $0.26(0.07)$ & $0.29(0.08)$ & $0.28(0.08)$ \\
\hline
\end{tabular}


Table 3

Average individual site accuracies of prediction of breeding values for parents and offspring from the non-combined nonspatial model for the full-sib (FS, FS model) and general combining ability tests (GCA, GCA model), the non-combined spatial model (GCA_sp model), and the combined non-spatial (GCA+FS model) and spatial (GCA+FS_sp model) models. See text for a complete description of the models.

\begin{tabular}{|c|c|c|c|c|c|c|c|c|c|c|c|}
\hline \multicolumn{12}{|c|}{ Accuracies of breeding values for parents } \\
\hline \multicolumn{6}{|l|}{ Series 1} & \multicolumn{6}{|l|}{ Series 2} \\
\hline \multirow{2}{*}{ SITE } & \multicolumn{5}{|l|}{ Model } & \multirow{2}{*}{ SITE } & \multicolumn{5}{|l|}{ Model } \\
\hline & FS & GCA & GCA_sp & GCA+FS & GCA+FS_sp & & FS & GCA & GCA_sp & GCA+FS & GCA+FS_sp \\
\hline $1 \mathrm{GCA}$ & - & 0.82 & 0.83 & 0.81 & 0.81 & $1 \mathrm{GCA}$ & - & 0.86 & 0.86 & 0.86 & 0.86 \\
\hline $2 \mathrm{GCA}$ & - & 0.88 & 0.89 & 0.89 & 0.89 & $2 \mathrm{GCA}$ & - & 0.87 & 0.87 & 0.87 & 0.88 \\
\hline $3 \mathrm{GCA}$ & - & 0.84 & 0.84 & 0.83 & 0.84 & $3 \mathrm{GCA}$ & - & 0.84 & 0.85 & 0.84 & 0.85 \\
\hline $4 \mathrm{GCA}$ & - & 0.84 & 0.84 & 0.83 & 0.84 & $4 \mathrm{GCA}$ & - & 0.86 & 0.86 & 0.86 & 0.86 \\
\hline $5 \mathrm{FS}$ & 0.77 & - & - & 0.85 & 0.85 & $5 \mathrm{FS}$ & 0.70 & - & - & 0.84 & 0.84 \\
\hline $6 \mathrm{FS}$ & 0.80 & - & - & 0.86 & 0.86 & $6 \mathrm{FS}$ & 0.64 & - & - & 0.81 & 0.81 \\
\hline Average & 0.79 & 0.85 & 0.85 & 0.85 & 0.85 & Average & 0.67 & 0.86 & 0.86 & 0.85 & 0.85 \\
\hline
\end{tabular}

\begin{tabular}{|c|c|c|c|c|c|c|c|c|c|c|c|}
\hline \multicolumn{12}{|c|}{ Accuracies of breeding values for offspring } \\
\hline \multicolumn{6}{|l|}{ Series 1} & \multicolumn{6}{|l|}{ Series 2} \\
\hline \multirow{2}{*}{ SITE } & \multicolumn{5}{|l|}{ Model } & \multirow{2}{*}{ SITE } & \multicolumn{5}{|c|}{ Model } \\
\hline & FS & GCA & GCA_sp & $\mathrm{GCA}+\mathrm{FS}$ & GCA+FS_sp & & FS & GCA & GCA_sp & $\mathrm{GCA}+\mathrm{FS}$ & GCA+FS_sp \\
\hline $1 \mathrm{GCA}$ & - & 0.51 & 0.52 & 0.51 & 0.52 & $1 \mathrm{GCA}$ & - & 0.62 & 0.62 & 0.64 & 0.64 \\
\hline $2 \mathrm{GCA}$ & - & 0.68 & 0.69 & 0.68 & 0.69 & $2 \mathrm{GCA}$ & - & 0.62 & 0.63 & 0.62 & 0.64 \\
\hline $3 \mathrm{GCA}$ & - & 0.57 & 0.57 & 0.57 & 0.57 & $3 \mathrm{GCA}$ & - & 0.57 & 0.57 & 0.58 & 0.58 \\
\hline $4 \mathrm{GCA}$ & - & 0.57 & 0.58 & 0.56 & 0.58 & $4 \mathrm{GCA}$ & - & 0.60 & 0.60 & 0.61 & 0.60 \\
\hline $5 \mathrm{FS}$ & 0.84 & - & - & 0.86 & 0.86 & $5 \mathrm{FS}$ & 0.86 & - & - & 0.87 & 0.86 \\
\hline $6 \mathrm{FS}$ & 0.85 & - & - & 0.85 & 0.85 & $6 \mathrm{FS}$ & 0.71 & - & - & 0.72 & 0.72 \\
\hline Average & 0.85 & 0.58 & 0.59 & 0.67 & 0.68 & Average & 0.78 & 0.60 & 0.61 & 0.67 & 0.68 \\
\hline
\end{tabular}

increased when the number of offspring by parents from the FS test increased for both the non-combined and combined analyses (Figure 1). However, the increase was more pronounced in Series 2. In our data, GCA and FS models showed lower accuracies of BVs for parents than GCA+FS model, due to the low accuracy of the FS parents breeding values calculated with the non-combined GCA or FS model. However, these differences decreased when the number of offspring added from the FS sites increase (Figure 2).

For offspring, the average accuracy of breeding values across the six complementary tests from the GCA+FS model was slightly smaller than those obtained from the GCA and FS models in Series 1, while this value was slightly higher in Series 2 (Table 3). However, these accuracies also varied between the GCA and FS tests. The average accuracy for offspring from the combined GCA+FS model were low, and increased for the GCA (average of $2.19 \%$ ) and FS (average of $0.93 \%$ ) tests of Series 2, and decreased for the GCA tests (average of $-0.86 \%$ ) and increase for the FS tests (average of $0.83 \%$ ) of Series 1, with respect to the non-combined GCA and FS models. Averaging across the two FS tests, the accuracies of BVs for the FS offspring showed no change with adding half-sib offspring from the four GCA tests in both FS tests (Figure 3). Moreover, slight differences in the accuracies of the FS offspring were observed in both sites between non-combined (FS model) and combined
(GCA+FS model) when we increased the number of half-sib offspring from the four GCA tests (Figure 3). However, these differences between the GCA+FS and FS models increased with an increasing number of half-sib offspring from the GCA tests (Figure 4).

The average accuracy of prediction breeding values across the six trials, calculated from the GCA+FS_sp model were slightly higher (only differences in the third decimal place) for parents and offspring, than corresponding values from GCA+FS model (Table 3). For the GCA_sp analysis, the average accuracy of prediction of breeding values were also slightly higher for parents and offspring, than corresponding values for parents and offspring calculated from GCA model (Table 3). These higher accuracies of breeding values for parents and offspring are associated with higher heritabilities estimated with spatial models for both combined and non-combined analyses (Table 2).

In summary, our results show that the additional information from the combined analyses resulted in negligible increases in accuracies of parents of the GCA compared to accuracies obtained by GCA analysis alone. In conctrast, for the parents of the FS tests, these increases were high (across both series higher than $7.87 \%$ ). Also, less than $1 \%$ increase in accuracies were found for the offspring of both FS and GCA tests. Similarity, increases in the accuracies for parents and offspring was less than $1 \%$ compared to the spatial and non-spatial models. It is 
Figure 1

Average of accuracies of breeding values for parents across the six complementary tests from the non-combined (GCA and FS models) and combined (GCA+FS model) analyses by the additional number of offspring by parents from the full-sib tests.
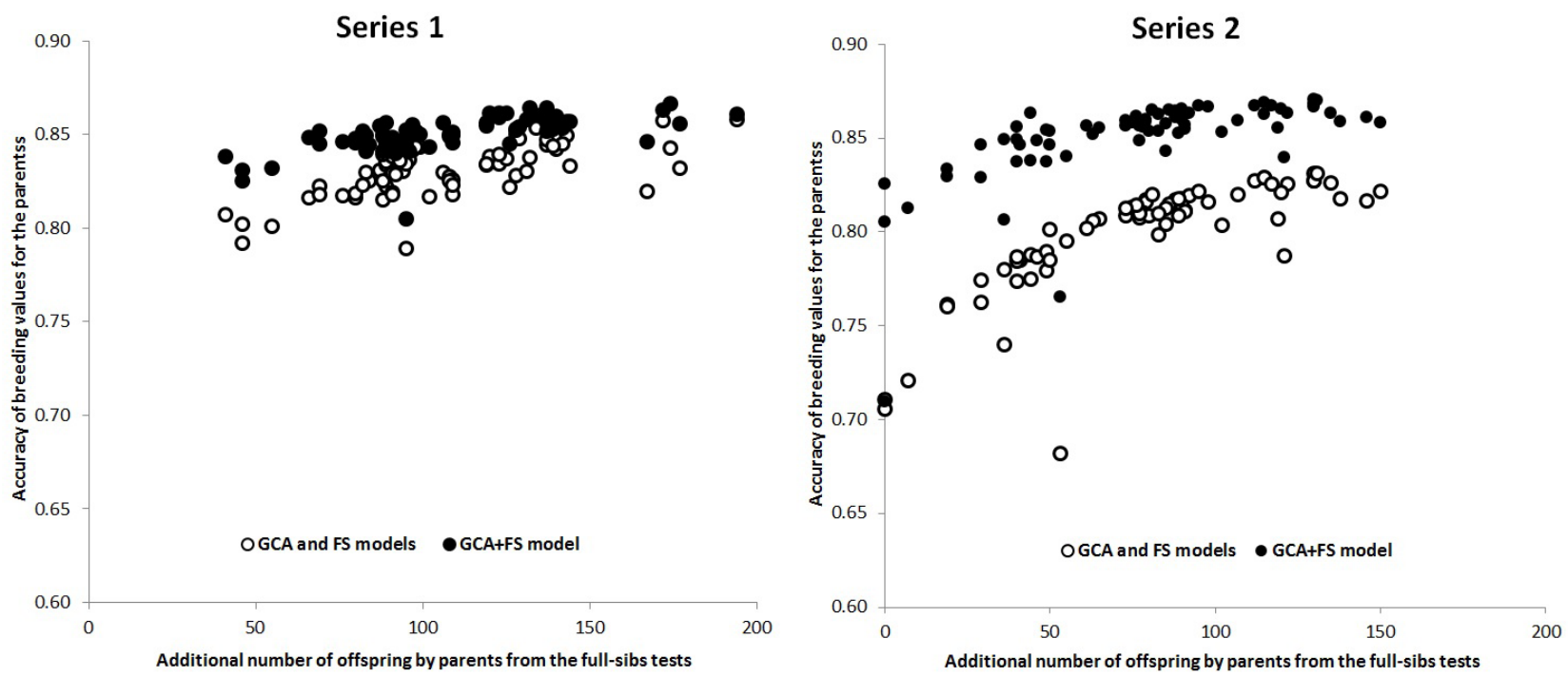

Figure 2

Percentage of increment in the average of accuracy of breeding values for parents across the six complementary test from the non-combined (GCA and FS models) and combined analyses (i.e., for the addition of offspring from the full-sibs tests, GCA+FS Model) by the additional number of offspring by parents from the full-sib tests.

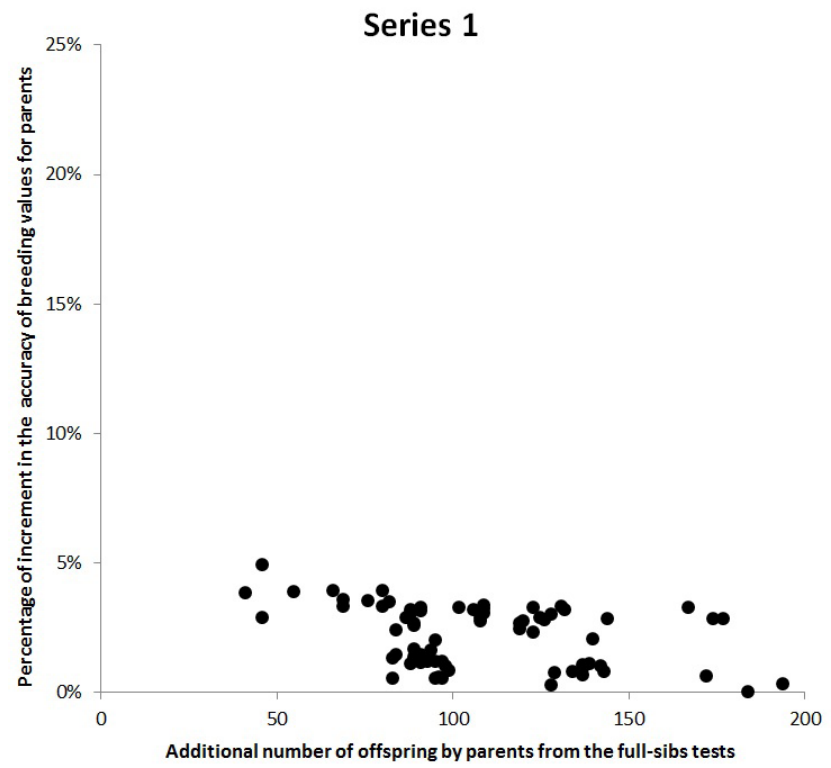

likely that the incomplete block (Alpha) design used for the four GCA tests already may have accounted for much of the local environmental heterogeneity (Cappa et al. 2013). This could explain why, on average, spatial analyses in both series were not very effective.

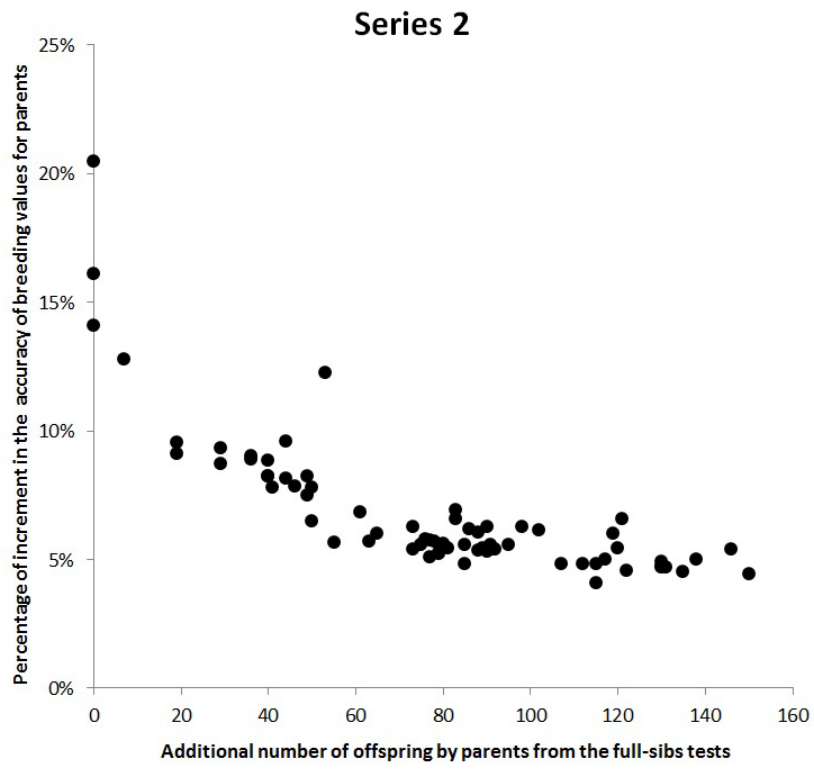

Overall, Spearman rank correlations between the noncombined and combined non-spatial (GCA+FS model vs. GCA and FS models) and spatial (GCA+FS_sp model vs. GCA_sp model) models were high in both series for predicted breeding values of parents and offspring (from 0.83 to 0.98 , results not shown), but for parents larger between the spatial models 
Figure 3

Average of accuracies of breeding values for offspring across the two full-sib tests from the non-combined (FS model) and combined (GCA+FS model) analyses by the additional number of half-sib offspring from the general combining ability tests.
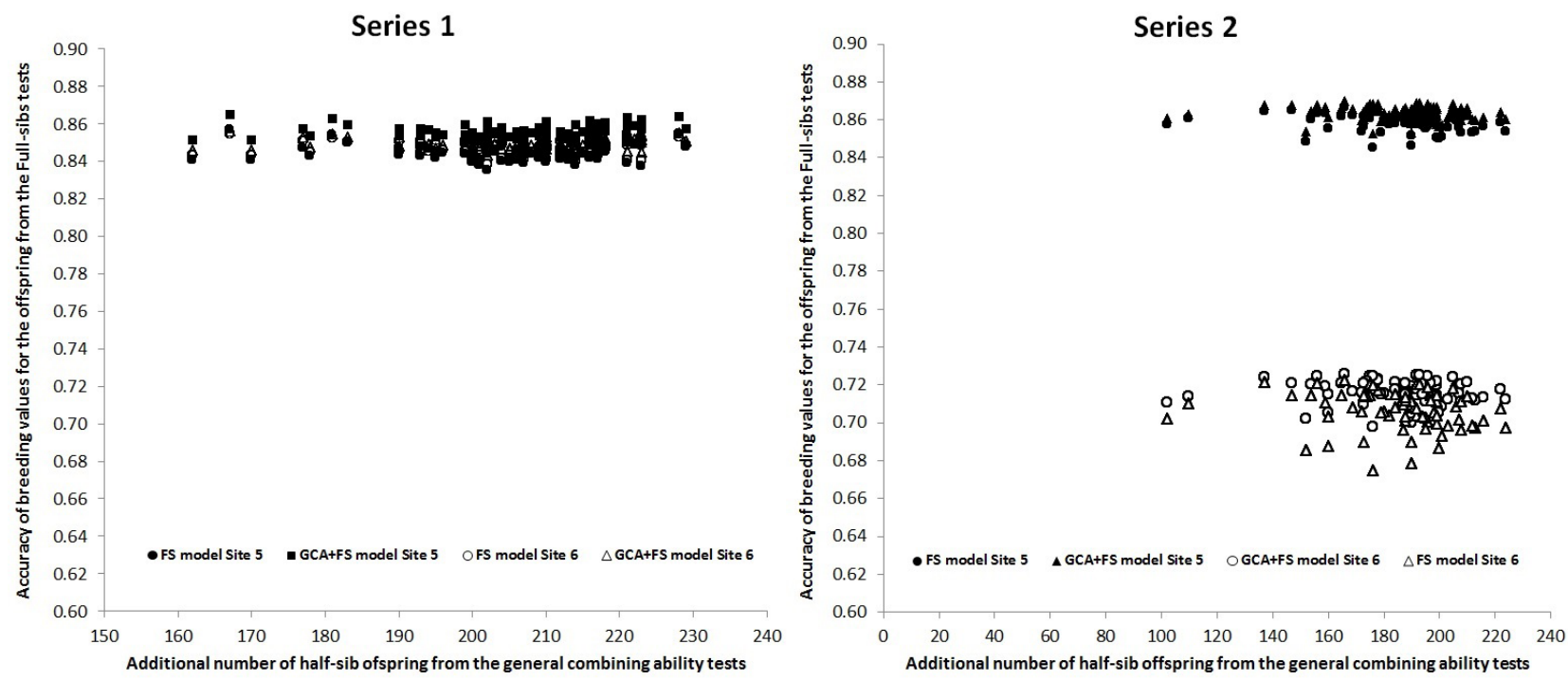

Figure 4

Percentage of increment in the average of accuracy of breeding values for full-sib offspring across the two full-sib test from the non-combined (FS model) and combined analyses (i.e., for the addition of offspring from the general combining ability progeny tests, GCA+FS model) and full-sib sites 5 and 6 by the number of half-sib offspring from the general combining ability tests.

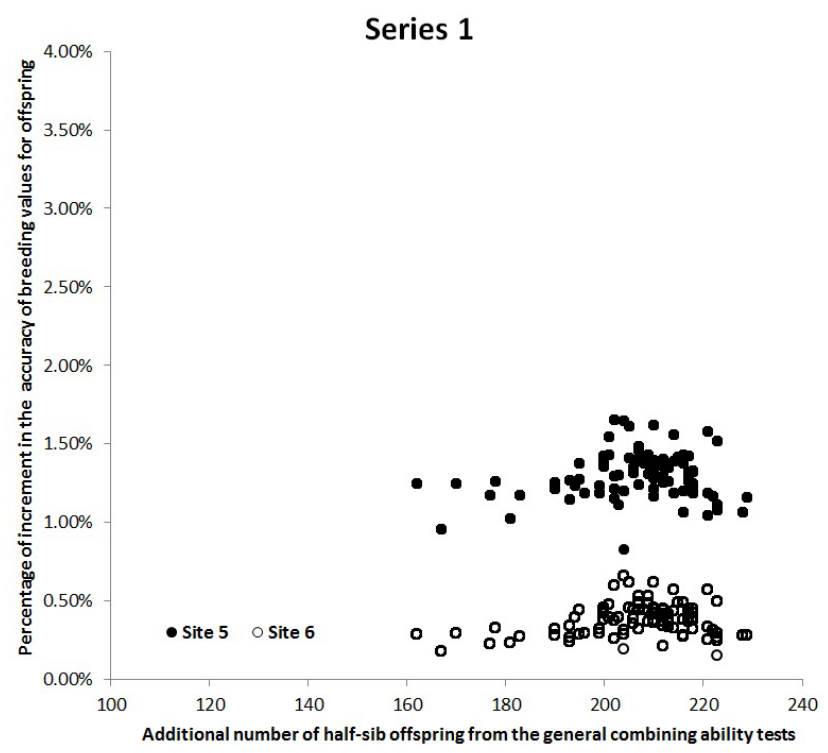

(average 0.90) than between the non-spatial models (average 0.88). Although Spearman rank correlations were high, there are some rank changes among the best 10 parents and 10 offspring across the mixed models studied in the two series (results not shown)

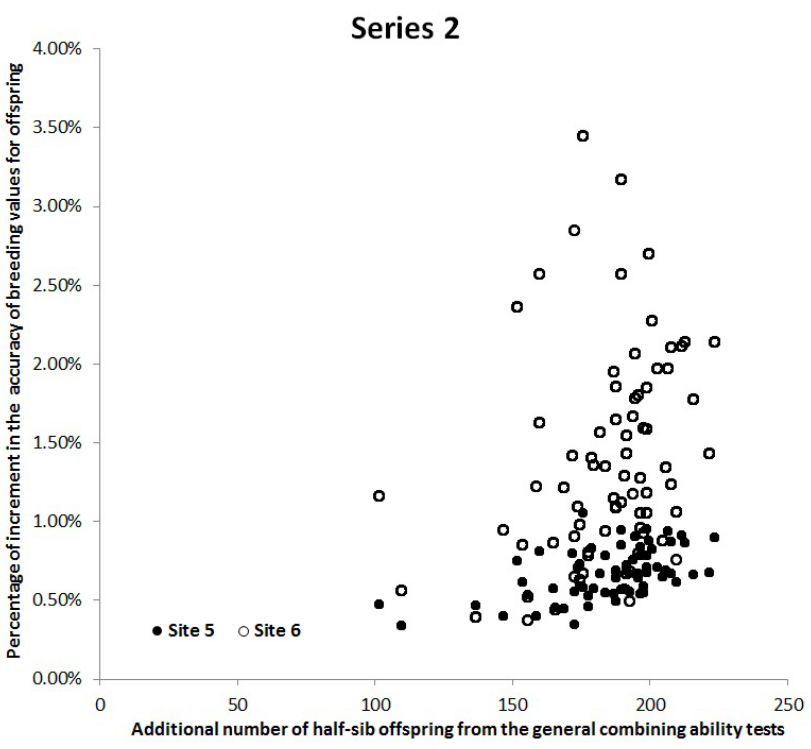

\section{Discussion}

Complementary mating designs allow the simultaneous ranking of parents for their general combining ability and to produce the next generation from within-family forward selection. 
To do this, polymix or GCA tests are planted in well-replicated single-tree plots using polymix-pollinated families; meanwhile, FS tests are planted in essentially un-replicated large block plots (from 25 to 100 trees) using control pollinated crosses between two parents. Complementary mating designs have been employed by several tree improvement programs (e.g. White et al. 1993; White et al. 1999; Johnson 1998b). However classical forward selections in complementary tests involve two steps (White et al. 2007, page 375): 1) the FS families are ranked based on the mid-parent BVs predicted from the polymix tests, 2) the best individual tree selections will then be made from within full-sib blocks. In this research, we applied a combined analysis of the polymix and FS tests using the mixed model approach to predict simultaneously BVs of grandparents, parents, full-sib families and forward selected offspring on the same scale. Moreover, a first order autoregressive spatial mixed model for the GCA tests was also implemented in the combined analysis. Combined analysis using the mixed model approach benefited from combining information between two generations from several genetic tests with varying mating and field designs. These abilities, coupled with the possibility to handle the spatial heterogeneity within trials, and the availability of genetic-test specific computer software, makes combined analysis a flexible tool for the analysis of complementary tests of forest trees.

Combined analysis using mixed model approach allowed estimates at the same time the BVs for parents, full-sib families and offspring of the six complementary progeny tests of coastal Douglas-fir program. Precise BV predictions are important in the GCA tests because these are used to select the appropriate parents to be used in crosses for the next generation. In our two series of Douglas-fir studied, the addition of two FS tests using the proposed combined analysis to increase the accuracies of the GCA parents BVs, was of little value (Table 3). These results highlighted that the number of progenies (average of 92) evaluated for parents in the four Douglas-fir GCA sites was adequate to obtain high accuracies (>0.82). Add more information from the two FS sites (i.e., more progenies with higher level of genetic relatedness) resulted only in slight increment on the accuracies of the parents BVs. We expect that the advantage of using combined analysis to increase the accuracy of parental BVs will be greater if there are smaller numbers of progenies available per parent from the GCA tests.

In the classical complementary tests, the FS tests are not used to rank parents (White et al. 1999). However, our empirical results show that the proposed combined analyses resulted in accuracies of the FS parents BVs comparable to the parents from the GCA sites (Table 3). While it is true that parental BV are generally obtained from GCA (polycross) tests, the added increase in accuracy derived from our combined analysis is still very important, as these parents are still producing seeds in seed orchards until they are replaced by their forward selected offspring. This can have immediate practical impacts as the best parents used in the GCA test under all likelihood are seed producing parents in current seed orchards. In Douglas-fir, it takes up to 15 years before new forward selections are mature enough to produce seed. In the meantime, the bulk of the seed produced is by the parents of these forward selections. Clearly, having more accurate parental BV is a large benefit especially in situations where orchard genetic gains are incorporated into annual allowable cut determinations as in British Columbia. These increases in the accuracies were higher for Series 2 than Series 1, suggesting that adding the four GCA sites have a higher impact on the parent accuracies when the number of progenies for parents was smaller in the FS sites (i.e., 5,566 vs. 7,832 for Series 2 and Series 1, respectively). Similar trends have been observed in two simulation data set when the previous generation was added (Johnson et al. 1998b; Ye et al. 2007). Johnson et al. (1998b) observed that when the first-generation data were added (i.e., data of the half-sib family from the firstgeneration tests from where the parents were selected), breeding values estimates of the parents showed very little improvement. Ye et al. (2007) evaluated the impact of using first-generation genetic information to increase selection efficiency in a second-generation breeding program. When parents were selected via forward selection, first-generation information provided little increase in the selection efficiency.

The additional information from parents could increase the ability to rank offspring for the subsequent generation. However, contribution of historical information depends on the quantity but also on the quality of the related genetic information (Ye et al. 2007). Then, the accuracy of breeding values, and hence the selection, can be influenced by the degree of genetic connectedness (Kennedy and Trus 1993), in our case, between the complementary tests. Combined analysis showed low increase of the accuracies of BVs for offspring (i.e., for forward selections) from the two FS sites, but was slight higher for Series $2(0.93 \%)$ than for Series 1 (0.83\%) (Table 3; Figure 2). Albeit a lot of progenies from the GCA tests were added for the forward selections (7,779 from Series 1 and 9,036 from Series 2 ), these progenies only add half-sib relationships. Thus, one reason for these results might be that the contribution of information from the GCA tests is lower (genetic covariance among half-sibs, 0.25 of the additive variance) than those that there was already within the FS tests (genetic covariance among fullsibs, 0.50 of the additive variance). Moreover, a large proportion of hidden relatedness from polycross parents of the GCA tests may affect the resulting accuracies of BVs for offspring from the two FS sites when the combined analysis is performed (El-Kassaby et al. 2011). Likely, Series 2 had a higher increase given the lower number of the FS progenies $(5,566)$ than Series 1 (7,832). Additionally, these results highlight that the number of offspring per cross generated and tested in the two FS tests (average by test 40.1) is adequate to obtain a high level of accuracies of these offspring (average within sites $>0.71$ ) in these Douglas-fir data sets. It seems likely that the advantage of using the combined analysis to improve the accuracies of FS offspring will be greater in data sets with smaller number of offspring per cross. Therefore, the Douglas-fir breeding program could reduce the testing population size in the future, or for the same number of test entries, use more sites.

The increase in accuracies also is dependent on the differences between the genetic correlations between the sites: the larger the differences in these correlations, the greater the gain 
in accuracy of evaluations (Mrode 2005). The correlation between all the six sites using the GCA+FS model was 0.66 and 0.69, for Series 1 and 2, respectively (see in Table 2); however, when different additive genetic covariance between pairs of test were fitted within each series (i.e., using an unstructured GA matrix), the average additive genetic correlation between GCA and FS sites was slightly lower, 0.59 (from 0.37 to 0.83 ) for the Series 1 and 0.69 (from 0.38 to 0.99 ) for Series 2. These results also could explain the low to moderate increment in accuracies of breeding values for backward and forward selections of the proposed combined analysis.

Spatial analysis, partitioning the residual variance into an independent component and a two-dimensional spatially autocorrelated component, is now routinely applied in forest genetic trails (e.g., Costa e Silva et al. 2001; Dutkowski et al. 2002; 2006). However, spatial MET analyses in data from forest genetic trial using this autoregressive covariance structure within of each trial is still limited (Ye and Jayawickrama 2008; Ding et al. 2008; Hardner et al. 2010). Consistent with these spatial MET studies, our empirical data set shows that combined (and non-combined) analyses jointly with spatial models gave an improvement (i.e., lower values of AIC) over the nonspatial combined (and non-combined) model, by accounting simultaneously for genotype by environment interaction and the presence of spatial heterogeneity within trials. However, these authors reported higher increases in accuracies of prediction of breeding values from the spatial MET model (from $3 \%$ to $17 \%$ ), than those found in our combined spatial GCA+FS_Sp model respect to the non-spatial GCA+FS model (averaging parents and offspring of $0.62 \%$ ), and non-combined spatial GCA_sp model respect to the non-spatial GCA model (averaging parents and offspring of $0.75 \%$ ).

\section{Conclusion}

Combined analysis using mixed model approach provides a flexible tool for the analysis of complementary tests. It can accommodate multiple generations of different genetic tests with different types of crosses, experimental and/or plot designs, and at the same time, account for the spatial heterogeneity within each trial by fitting spatial models through a first-order autoregressive residual covariance structure. Douglas-fir for a single variable shows that additional information provided by the proposed combined analysis produced an improvement in model fit when compared with the non-combined analysis. The improvements in the accuracies of breeding values for backward and forward selections were generally modest, but in general, in favour of the proposed combined analysis. Spatial combined analysis gave slightly better results than the non-spatial combined model. The combined analysis approach will be most effective when the number of offspring per GCA parents and/or per full-sib cross be lower than those used for the analysis of this empirical Coastal Douglas-fir data set.

\section{Referenences}

Akaike $\mathrm{H}$ (1974) A new look at the statistical model identification. IEEE Trans Automat Contr 19:716-723. Available at http://dx.doi.org/10.1109/ tac.1974.1100705

Cappa EP, Yanchuk AD, Cartwright CV (2013) Bayesian inference for multi-environment spatial individual-tree models with additive and full-sib family genetic effects for large forest genetic trials. Ann For Sci 69:627-640. Available at http://dx.doi.org/10.1007/s13595-011-0179-7

Costa e Silva J, Dutkowski GW, Gilmour AR (2001) Analysis of early tree height in forest genetic trials is enhanced by including a spatially correlated residual. Can J For Res 31:1887-1893. Available at http://dx.doi.org/10.1139/cifr-3111-1887

Costa e Silva J, Potts BM, Dutkowski GW (2006) Genotype by environment interaction for growth of Eucalyptus globulus in Australia. Tree Genet Genomes 2:61-75. Available at http://dx.doi.org/10.1007/s11295-005-0025-x

Ding M,Tier B, Dutkowski GW (2008) Multi-environment trial analysis for Pinus radiata. New Zeal J For Sci 38:143-159

Dutkowski GW, Costa e Silva J, Gilmour AR, Lopez GA (2002) Spatial analysis methods for forest genetics trials. Can J For Res 32:2201-2214. Available at http://dx.doi.org/10.1139/x02-111

Dutkowski GW, Costa e Silva J, Gilmour AR, Wellendorf H, Aguiar A (2006) Spatia analysis enhances modeling of a wide variety of traits in forest genetic trials. Can J For Res 36:1851-1870. Available at http://dx.doi.org/10.1139/x06059

El-Kassaby YA, Cappa EP, Liewlaksaneeyanawin C, Klapste J, Lstiburek M (2011) Breeding without Breeding: Is a complete pedigree necessary for efficient breeding? PLoS ONE 6(10): e25737. Available at http://dx.doi.org/10.1371/ journal.pone.0025737

Gilmour AR, Culli BR, Verbyla AP (1997) Accounting for natural and extraneous variation in the analysis of field experiments. J Agr Biol Envir St 2:269-293. Available at http://dx.doi.org/10.2307/1400446

Gilmour AR, Gogel BJ, Cullis BR, Thompson R (2009) ASRemI User Guide Release 3.0 VSN International Ltd, Hemel Hempstead, HP1 1ES, UK. www.vsni.co.uk

Hardner CM, Dieters M, Dale G, DeLacy I, Basford KE (2010) Patterns of genotype-by-environment interaction in diameter at breast height at age 3 for eucalypt hybrid clones grown for reafforestation of lands affected by salinity. Tree Genet Genomes 6:833-851. Available at http://dx.doi.org/10.1007/ s11295-010-0295-9

Heaman JC, Woods JH (1989) The coastal Douglas-fir breeding program in British Columbia. Breeding Prog. Rev. Rep. Res. Br. Ministry of Forests, Victoria, BC. $42 \mathrm{p}$.

Henderson CR (1984) Applications of linear models in animal breeding. Canada, University of Guelph, Guelph, Ontario. 439 p.

Johnson GR (1998a) Parental GCA testing: how many crosses per parent. Can J For Res 28:540-545. Available at http://dx.doi.org/10.1139/cjfr-28-4-540

Johnson GR (1998b) Breeding design considerations for coastal Douglas-fir. USDA For. Ser. Gen. Tech. Rep. PNW-GTR-411. 40 p. Available at http://dx. doi.org/10.2737/pnw-gtr-411

Kennedy BW, Trus D (1993) Considerations on genetic connectedness between management units under an animal model. J Anim Sci 71:2341-52

Mrode RA (2005) Linear models for the prediction of animal breeding values. CAB International, Wallingford, Oxon OX10 8DE, United Kingdom, 2 edition. $344 \mathrm{p}$.

Namkoong G (1979) Introduction to quantitative genetics in forestry. USDA For. Serv. Tech. Bull. 1588. 342 p.

Stoehr MU, Yanchuk AD, Xie CY, Sanchez L (2008) Gain and diversity in advanced generation coastal Douglas-fir selections for seed production populations. Tree Genet Genomes 4:193-200. Available at http://dx.doi.org/10.1007/ s11295-007-0100-6

Whitacker D, Williams ER, John JA (2002) CycDesigN version 2 software. CSIRO Forestry and Forest Products, Australia. $41 \mathrm{p}$.

White TL, Hodge GR, Powell GL (1993) Advanced-generation tree improvement plan for slash pine in the southeastern United States. Silvae Genet 42:359371

White TL, Matheson AC, Cotterill PP, Johnson RG, Rout AF, Boomsma DB (1999) A nucleus breeding plan for radiata pine in Australia. Silvae Genet 48:122-133 
White TL, Adams WT, Neale DB (2007) Forest genetics. CABI Publishing. 682 p. Ye TZ, Jayawickrama KJS, Johnson GR (2007) Efficiency of including first-generation information in second-generation ranking and selection: results of computer simulation. Tree Genet. Genomes 3:319-328. Available at http:// dx.doi.org/10.1007/s11295-006-0071-z

Ye TZ, Jayawickrama KJS (2008) Efficiency of using spatial analysis in firest-generation coastal Douglas-fir progeny tests in the US Pacific Northwest. Tree Genet Genomes 4:677-692. Available at http://dx.doi.org/10.1007/s11295008-0142-4 